\title{
Robust learning of arm trajectories through human demonstration
}

\author{
Aude Billard \& Stefan Schaal \\ Computer Science and Neuroscience, HNB-103, Univ. of Southern California, Los Angeles, CA 90089-2520 \\ Kawato Dynamic Brain Project (ERATO/JST), 2-2 Hikaridai, Seika-cho, Soraku-gun, 619-02 Kyoto, Japan \\ \{billard,sschaal\}@usc.edu
}

\begin{abstract}
We present a model, composed of hierarchy of artificial neural networks, for robot learning by demonstration. The model is implemented in a dynamic simulation of a 41 degrees of freedom humanoid for reproducing $3 \mathrm{D} \mathrm{hu}$ man motion of the arm. Results show that the model requires few information about the desired trajectory and learns on-line the relevant features of movement. It can generalize across a small set of data to produce a qualitatively good reproduction of the demonstrated trajectory. Finally, it is shown that reproduction of the trajectory after learning is robust against perturbations.
\end{abstract}

\section{Introduction}

Robot teaching by demonstration makes an increasing body of robotics research. A large part of these efforts focus on assembly task-learning. Movements of a human performing object moving/stacking tasks are recorded, either using video images $[1,2]$ or using a manipulandum, for measuring directly the joint torques $[3,4]$. Data are then analyzed to determine the torques required for an industrial robot arm to reproduce the motions with similar precision. A major issue in robot teaching by demonstration is to define an algorithm which can remove inconsistencies across the demonstrations, while keeping enough information to allow reproduction of fine features of the movement [5]. As the level of precision of data segmentation is highly taskdependent, it requires the development of adaptive and learning algorithms [6].

Human ability to imitate oustands that of all other animals [7]. Human imitation ranges from gross reproduction of general body postures (pantomine) to precise reproduction of joint motions (as in dance, surgery, etc). Recent trends in robotics takes inspiration in studies of human imitation to develop architectures for visuo-motor control and learning in robots which would show some of the flexibility of natural systems $[8,9,10,2,11,12]$. In $[9,13]$, we developed a biologically plausible model of human imitation. In this paper, we discuss the potential of this model for controlling a 3 degrees of freedom robot arm.

\section{The model}

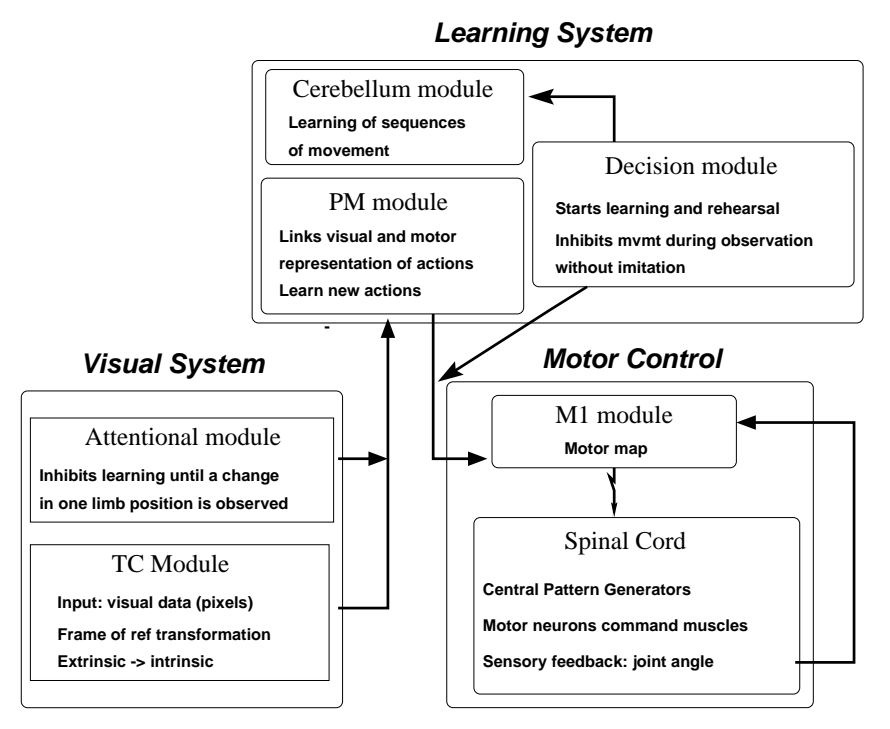

Figure 1: The architecture consists of seven modules which give an abstract and high-level representation of corresponding brain areas involved in visuo-motor processing. The seven modules are: the attentional and temporal cortex modules, the primary motor cortex and spinal cord modules, the premotor cortex and cerebellum module, and the decision module.

This work builds upon our general model of learning by imitation $([9,13])$, see Figure 1 . The model is biologically inspired in its function, as its composite modules have functionalities similar to that of specific brain regions, and in its structure, as the modules are composed of artificial neural architectures. It is loosely based on neurological findings in primates and incorporates abstract models of some brain areas involved in visuomotor control, namely the temporal cortex (TC), the spinal cord, the primary motor cortex (M1), the premotor area (PM) and the cerebellum.

In this paper, we briefly describe the parts of the model important for the specific implementation. The reader can refer to [9] for a detailed description. 


\subsection{Data privegss}

The visual module performs four levels of processing of the datia) transformation of frame of reference, from extrinsic to intrinsic, 2) filtering of small and noisy motions, 3) segmentation of the motion, based on changes in velocity and movement direction, and 4) parameterization of the movements in terms of speed and direction. In the experiments reported in this paper, see Section 3, data are recorded in joint angles, and, thus, step 1 is not performed.

\section{Filtering of small motions}

Input data to this module are all recorded joint angular trajectories of arm movement. We apply a threshold function (see Equation 1) to the position trajectory of each degree of freedom (DOF) to eliminate small movements, due to the interaction torques across the body. These small movements are noise to us, as we wish to recognize and model only the key features of voluntary movements.

Let $\left.\not t_{i}\right)$ be the angular displacementstifijeoint

t. $D_{i}^{M}, D_{i}^{m}$ and $D_{\text {pare the maximal, minimal and mean }}$ values of $i$ over the whole trial. The high-pass filter eliminates from further processing into segmentation (see below) all joint trajedthenesh are such that

$$
D_{j}^{M}-D_{j}^{m}<\frac{p i}{16}
$$

\section{Segmentation}

Data are segmented to detect the starts and stops of voluntary motion and changes in direction of movement, following Equations 2, 3 and 4. The segmentation process depends on a set of 3 parameters per DOF. These are the minimeifh displacement ${ }_{j}$ (in joint angle) for detecting a motion, the minimum time windowTduring which no displacem $\oplus$ int greater than has been observed. Siffecedit degrees of freedom havefftirent dynamic properties,fflerento their di lengths and muscular composifferente applied di segmentation parameters to each. These properties are calculated as follows.

$$
\begin{gathered}
\theta_{j}^{m}=8 \cdot \frac{p i}{D_{j}^{M}-D_{j}^{m}} \\
\theta_{j}^{M}=2 \cdot \theta_{j}^{m} \\
T_{0}=2 \cdot T \text { with T such that } \quad{ }_{i}(T)=\tilde{D}_{i}
\end{gathered}
$$

\section{Parameterization}

After segmentation, the speed and direction of movement of each joint is coded in the output of the TC neurons to the PM neurons There are two neurons per degree of freedom (DOF) per joint, coding for positive and negative direction of movement, respectively. Let $y_{i}^{T C}(t)$ be the output of $\mathrm{kat}_{\mathrm{C}}$ nidquarod the series of time steps at which the segmentation has occurred, then

$$
y_{i}^{T C}\left(t_{j}\right)=D_{i}\left(t_{k}\right)-D_{i}\left(t_{k-1}\right)
$$

and $\mathrm{k}$ is sudh that $=t_{k}$ angl $>1$.

Figure 2 shows segmentation of the trajectory of the shoulder joints (flexor/extensor, abduction/adduction) of the left arm during drawing of a figure eight. Figure 2 shows the speed input coded by the neurons after segmentation.
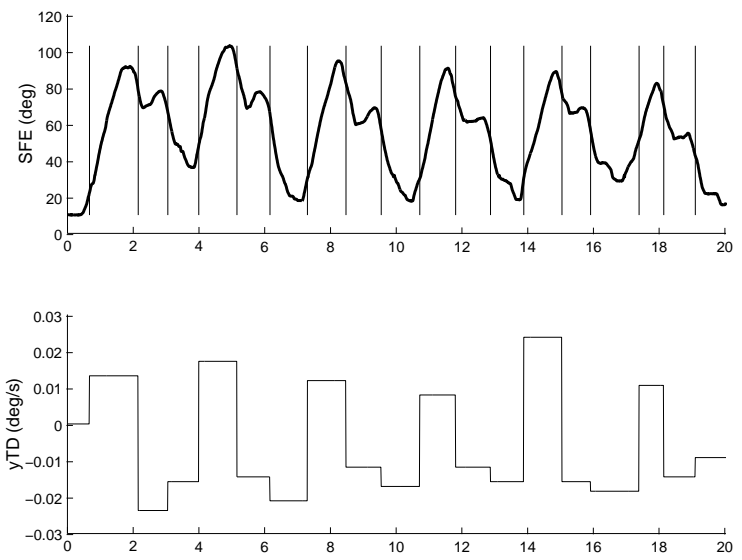

Figure 2: Top: Trajectory of shoulder oi $j$ nt ( $\mathrm{SFE}=$ flexion/extension) during drawing of a gfire eight. Vertical bars show the time steps of segmentation. (Bottom) Output of the neurons fol lowing segmentation. The output is proportional to the value of the speed of the motion at the time of the segmentation.

\subsection{Motontreb}

In our model, motor control is hierarchical and is composed of (from lowest level to highostuskeel) modełpinal c,omdotor cortex (M1), premotor cortex (PM) and cerebellum modules.

\section{Spinal cord module}

The module receives excitatory input from the M1 neurons (defining the amplitude and speed of the movement for each DOF) and outputs to the muscle model which then computes joint torques sent to the dynamic simulation. It is composed of fixed neural circuits, one for each DOF for each joint, which produce an oscillation of that joint, following [14].

The neurons of the spinal cord module are modeled as leaky-integrators, which compute the average firing frequency [15]. According to this model, the mean membrane potemtiofl a neuñoceiving input from M1 noclis governed by the equation

$$
\tau_{i} \cdot d m_{i} / d t=-m_{i}+\sum w_{i, j} x_{j}
$$


where $_{j}=\left(1+e^{\left(m_{j}+b_{j}\right)}\right)^{-1}$ represents the 'neuron $\mathrm{s}$ short-term average firingb fireqhennuron

$\mathrm{s}$

bias, $\tau_{i}$ is a time constant associated with the passive properties of the neuron s membrane, and $w_{i, j}$ is the synaptic weight of a connectiøh from neuron $j$ to neuron $N_{i}$. In the model, a neuron in the spinal cord receives input from all other neurons and from neurons in M1 module.

Muscle torques Each DOF of each joint is controlled by a pair of muscles which receive input from corresponding pair of motor neurons in the spinal cord module. A muscle is simulated as a combination of a spring and a damper [16]. The torque exerted on each joint is determined by a pair of opposed flexor and extensor muscles. These muscles can be contracted by input signals from motor neurons, which increase their spring constant, and therefore reduce their resting length. The torque acting at a particular joint is therefore determined by the motoneuron alctivities ( and $M_{\ell} f$ the opposed flexor and:extensor muscles

$$
T=\alpha\left(M_{f}-M_{e}+\beta\left(M_{f}+M_{e}+\gamma\right) \Delta \varphi+\delta \Delta \dot{\varphi}\right.
$$

where $\Delta$ is tffiereelice between the actual angle of the joint and the angle at rest (zero in our experiments). Flarent ffocients $\quad \alpha, \beta, \gamma$, and $\delta$ determine, respectively, the gefimes \$lgaiti the tonic stiffness, and the deffiriontgofdthe muscles.

\section{M1 and PM modules}

M1 module conttimsofatipe

body. It is composed of 3-neuron networks for each DOF of each joint for independently regulating the amplitude (two nodes) and the frequency (one node) of the oscillation of the corresponding joint DOF, similar to [14].

The PM module creates a direct mapping between the parameterization of the observed movement in TC, following visual segmentation, and that used for motor control in M1. PM nodes receive sensory feedback, in terms of joint angle position, from the spinal cord module. $y_{j}^{S}(t)$ is the angle of degreegifflifmeedom $\mathrm{b} l$. The term $\delta\left(y_{l_{j}}^{S}, y_{l_{k}}^{T C} \cdot(\pi / 8)\right)$ in equation 2.2 compares the actual deviationirofthinivatar and the

deviation of the human arm, given by $y_{l_{k}}^{T C}$ for corresponding direction d6finoinon $\quad 1$. This term modulates the amplitude or speed of the movement, by increasing or decreasing (for smaller or larger speed) the output of the PM and consecutively of the M1 nodes (and finally the torque sent to the motor neurons). The network TC-PM-M1 consists of a Dynamical Recurrent Associative Memory Architecture (DRAMA) [17]. Similarly to time delay networks [18], each connection is associated with two parameters, a weight $w_{i j}$

\footnotetext{
${ }^{1}$ The term $y_{l_{k}}^{T C}$ is a measure of the speed at the beginning of the movement. We map it into a measure of position by multiplication with the factor $(\pi / 8)$.
}

and a time parameter $\tau_{i j}$. Weights correspond to the synaptic strength, while the time parameter specifies a synaptic delay, that is a delay on the time required to propagate the activity from one neuron to the other. It is a fully connected network (all nodes in PM are connected to all nodes in TC and to all nodes in M1, nodes in TC and M1 are not interconnected) with asymmetrical connections $\left(w_{i j}=w_{j i}\right)$ and self-connections on each unit.

If $(\tilde{w}, \tilde{\tau})$ and $(w$, tate are the weights of the connections between M1 and PM, and between TC and PM modules respectively, if $y_{k}^{T C}$ is the output of TC neuron for direction of kofoliement $\mathrm{b} l$, then $y_{j}^{M 1}$, the output of M1 neuron of di்giner of freedom $l$ is:

$$
\begin{array}{r}
d y_{l_{j}}^{M 1} / d t=-\tilde{\tau}_{l_{j} l_{j}}^{M 1}+ \\
\sum_{i} \tilde{w}_{i l_{j}} \cdot y_{i}^{P M} \cdot \delta\left(\tilde{\tau}_{i l_{j}}, y_{i}^{P M}\right) \cdot \delta\left(y_{l_{j}}^{S}, y_{l_{k}}^{T C} \cdot \frac{\pi}{8}\right)
\end{array}
$$

and $y_{j}^{P M}$, the output of fineumordule PM

$$
\begin{array}{r}
d y_{l_{j}}^{P M} / d t=-\tau_{l_{j} l_{j}}^{P M}+ \\
\sum_{i} w_{i l_{k}} \cdot x_{i}^{T C} \cdot \delta\left(\tau i l_{k}, y_{i}^{T C}\right) \cdot \delta\left(y_{l_{j}}^{S}, y_{l_{k}}^{T C} \cdot \frac{\pi}{8}\right)
\end{array}
$$

The function $\delta(x, H)$ is a threshold function that outputs 1 waten $\quad=H$ and 0 otherwise.

The sets of weights $\tilde{w}$ and $w$ define the mapping between the visual representation and the torques sent to the muscles. The relationship $\tilde{w}=f(w)$ is a first order approximation of the inverse dynamics calculation. In previous experiments, all connection parameters $(w)$ and temporal $(\tau)$ were modulated by learning, see [9]. In the experiments reported here, this mapping is fixed and follows a first order relationship, such that for activation of degree of fifelidom $\quad \mathrm{b} l$, represented by activity in nejumol, nim and neuron

$k$ in $\mathrm{TC}$, we have

$$
\tilde{w}_{j i}(t)=I_{l_{j}}(t) \cdot w_{i l_{k}}
$$

where $I_{l_{j}}(t)$ is the moment of inertia of the limb $l$ using DOF The moment of inertia varies depending on the position of the limb attached to this limb. Here we consider only the shoulder/elbow complex. In a first approximation, we estimate

$$
\begin{gathered}
I_{l_{k}}(t)=I_{l_{j}}\left(t_{0}\right), l=0,1 \\
I_{l_{k}}(t)=I_{l_{j}}\left(t_{0}\right) \cdot\left(D(t) \quad-D\left(t_{0}\right)\right), \quad l=2
\end{gathered}
$$

$t_{0}$ is the time at which the arm is in the relaxed position (aligned along the body). $l=0,1,2$ are the 3 DOFs (SFE, SAA, SHR) of theisshbellderFanfl the elbow. 


\section{Cerebellum and Decision modules}

Learning of the complete movement is done in the cerebellum module. This module is a DRAMA architecture. It receives input from the PM module and learns the time series of activity of neurons in PM, which represent the sequence of motions after segmentation. Learning consists of updating the parameters of the connection between cerebellum and PM modules ( $w$ and $\tau$ ) following Hebbian rules, given by Equations 13 and 14 .

$$
\begin{gathered}
\delta w_{j i}(t)=a \cdot y_{i}(t) \cdot y_{j}(t) \\
\tau_{j i}(t)=\left(\frac{\tau_{j i}(t-1) \cdot \frac{w_{j i}}{a}+\frac{y_{j}(t)}{y_{i}(t)}}{\frac{w_{j i}}{a}+1}\right) \cdot y_{i}(t) \cdot y_{j}(t)
\end{gathered}
$$

whereis a constant factor by which the weights are incremented.

Finally, the decision module controls the transition between observing and reproducing the motor sequences, i.e., it inhibits PM neural activity due to TC (visual) input to flow downwards to M1 (for motor activation). It is implemented as a set of if-then rules and has no direct biological inspiration.

\section{3-D biomdenial simbationfo a hu- maid}

The model is implemented in a dynamic simulation of a 41 degrees of freedom (DOF) avatar [19] (see Figure 3 right). Shoulders, hips, wrists, ankles and head have 3 DOFs. Elbows and knee have one. The trunk is made of three segments with 2 DOFs each. All limbs are attached by hinge joints. The external force applied to each joint is gravity. Balance is handled by supporting the;hiposund contact is not modeled. There is no collision avoidance module. The dynamics model is derived from the Newton-Euler formulation of Rigid Body Dynamics.

\section{Experiments}

Data for these experiments are recordings of human arm motion, gathered using a Sarcos SenSuit (see Figure 3 left). The complete SenSuit is worn like an exoskeleton which, for most movements, does not restrict the motion while an array of lifferttnseight Hall-e

sors reliably records the positions of 35 degrees of freedom (DOF) of the human body. In the experiments, we used only recordings of the 3 degrees of freedom (DOF) of the shoulder joint (flexion, abduction and humeral rotation) and of the elbow joint. Data are captured at a frame rate of $100 \mathrm{~Hz}$.

Because of space constraints, we present here the model s implementation on one set of data. These are recordings of left arm movement for five repetitions of drawing a figure eight. Data were segmented following the algorithm of Section 2.1, as shown in Figure 2. The model was presented with the whole trajectory once

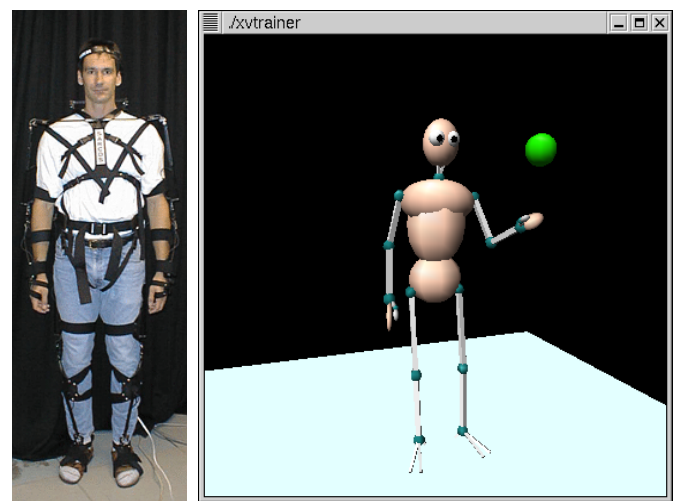

Figure (Left) Subject weari ng the Sarcos sensuit recording system. (Right) Simulation of A 1 degrees of freedom humanoid.

(composed of 6 repetitions of the complete movement). The amplitude and speed of the movement varies over the repetitions. The model was let reproduce the trajectory both during training and after learning. Figure 5 shows superimposed human subject and avatar trajectories of shoulder $(\mathrm{SFE}=$ flexor/extensor, $\mathrm{SAA}=$ abduction/adduction) and elbow (EB) joints during training. Figure 4 shows the values of the torques sent to the same 3 DOFs of the avatar during the training phase. In this example, only these 3 DOFs received active control for reproducing a desired trajectory (after segmentation). The remaining joints of the arm and of the rest of the body were kept immobile, receiving torques to cancel the internal perturbations due to the motions of other joints and (i.e. $y^{T C}=0$ for these joints).
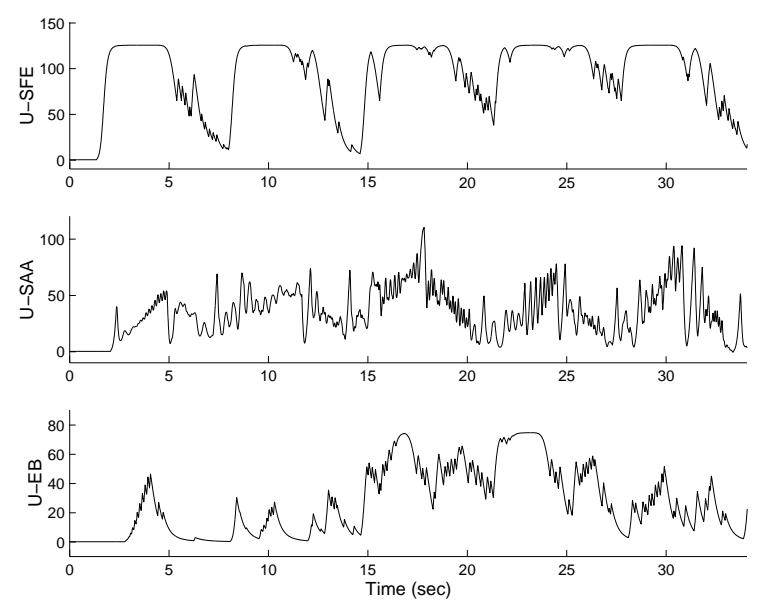

Figure 4: Torque sent to the shoulder joints, (SFE=flexor/extenso), (SAA abduction/adduction) and elbowoji nt (EB) during the reproduction of fure ei ght with the left arm. 

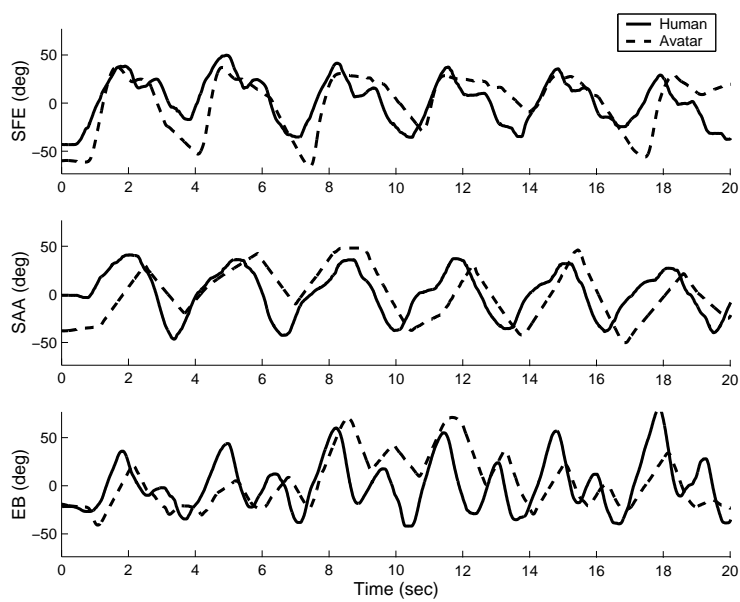

Figur 5 Trjectory of the shoul derjoi nts, (SFE=flexor/extenso), (SAA abduction/adduction)and el bowgi nt (EB) during the reproduction of eifure ei ght with the left arm.

Results show that the system follows closely the demonstrated trajectory during training, mapping the changes in amplitude and speed. The model manages to extract on-line the main features of the movement, while dismissing most of the (from our point of view) unimportant fine structure of the movement and noise (from the recording system). Figure 6 shows the increase of neural firing during training. After two presentations of the movement, the network has generalized. That is, it has learned to recognize the four main features of the movement and to represent those with a specific neural firingepatmen node1-node2-

node3-node2. Note that the nodes code for the combined movement of the 3 DOFs (SFE/SAA/EB) and not for each joint separately. For clarity of the figure, we show only the SFE joint superimposed to the neural firing rate.

The same pattern of node firing is activated during the 4 remaining presentations of the movement, showing that the network recognizes correctly the movement. During training on the 4 last movements, the network adjusts the parameters (the $\tau_{i j}$ from equation 14) of these nodatserconnections to better represent the precedence and time lag between each sub-movement. After training, the model produce a movement which presents the main sub-features of the training movement (see Figures 7 and 8). Amplitude and speed of this reproduced movement represents the weighted combination of that of the six demonstrations.

Robustness in the face of perturbation is an important criteria for a robotics system. We tested the model s robustness after training, by applying an external force during rehearsal of the trajectory. Figures 7 and 8 show the trajectory of SFE and EB DOFs when applying a vertical force on the wrist of 200 and 1200 Newton respectively. Results show that the system re- covers quickly to both constraintsffectthiis due to the PID control term $\left(\delta\left(y_{l_{j}}^{S}, y_{l_{k}}^{T C} \cdot(\pi / 8)\right)\right)$ of equation 2.2. The desired trajectory $\left(y_{l_{k}}^{T C}\right)$ is obtained by rehearsal of the activity of neurons in the TC module (feeding back the activity from PM neurons).
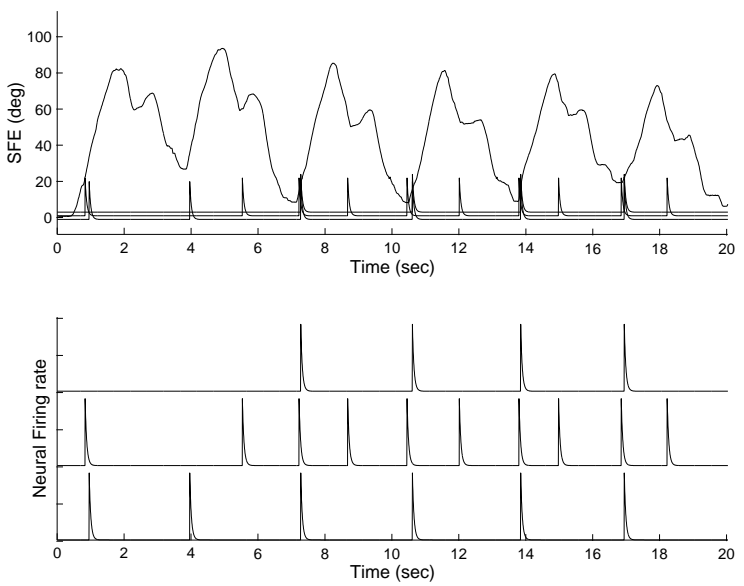

Figur6 Firing rate of the neurons in the cerebellum module during training.
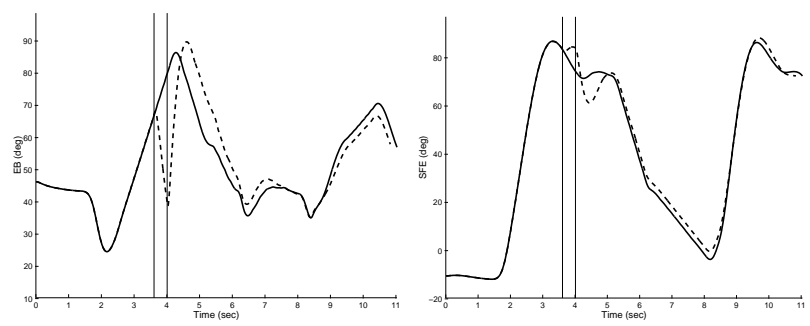

Figure Tỷactory of the shoul der ji nts ( $\mathrm{SFE}=$ flexor/extenso) and elbowgi nt (EB) during rehearsal after training. Solid line: desired trjectory, dashed 1 ine: trjectory after perturbati on (vertical force of $0 \mathrm{Kg}$ ) lasting 0.2 seconds.

\section{Conclusion}

This paper presented a biologically inspired model of human imitation. The model was implemented in a dynamic simulation of a humanoid avatar with 41 degrees of freedom for reproducing 3D human arm motion. It was shown that the model could learn the principal features of a 3 DOFs arm trajectory, by generalizing across tffferdint demonstrations. Learning is fast and done on-line (here on a pentium III, $700 \mathrm{MHz}$ station). In [17], we showed that the DRAMA architecture allowed on-line learning and reproduction of movements in robots with very limited computational power (124M 

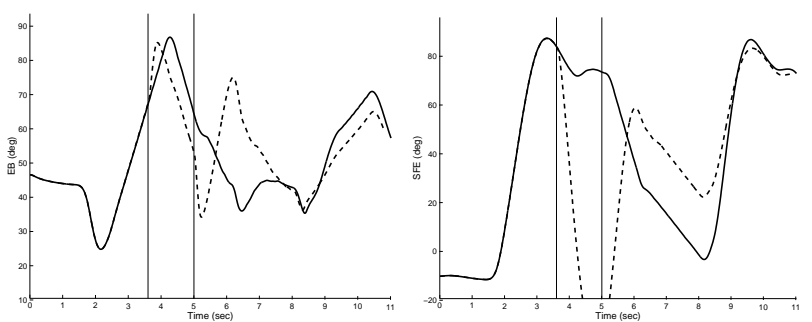

Figur 8 Tijectory of the shoul derjoi nts, ( $\mathrm{SFE}=$ flexor/extenso) and elbowöi nt (EB) during rehearsal after training. Solid line: desired trjectory, dashed 1 ine: trjectory after perturbati on (vertical force of $120 \mathrm{Kg}$ ) lasting 0.7 seconds.

RAM, $30 \mathrm{MHz})$. Further, it was shown that reconstruction of the movement was robust against perturbation. Generalization, on-line learning and robustness against perturbation are desirable for a robot controller. However, a number of questions remain to be answered in order to determine the usefulness of our model to control a robot:

1) Scaling up to full body matiblthough we have shown that the model could well reproduce motion of a 65 degrees of freedom humanoid when given simulated (not noisy) data [9], it remains to show whether the performance of the model would degrade when controlling actively (as opteresponse to

internal torques) more joints than the 3 used here and for a more general set of data.

2) Range of validity. of luherenopletiments

showed that a qualitatively good reproduction of the features of the movement can be obtained by giving only the following informatioli) thhehe system starts and stops of the movefficientatiod it is su determine those within a Grefishiendufra 0

tion of the mo,rentmetinitial speed at each start point of the mornednata first order approxima-

tion of the inverse dynamics. It remains now to determine the range of motions within which the above set of minimal constraints holds. The present experiments showed only thfitieientiforu 3D slow motions of the arm, when the torso is in upright position and when only 3 joints are active, the other joints being kept immobile.

\section{Aknwegh ents}

This wdx was made possi ble by Awards\#97 10312 and\# 0082995 of theU S National Science Foundation, the ERATO Kawato hynamic Brain Poject funded by Jlpenese Science and Technology Cooperation, and the ATR Humanformati on Pocessi ng Research Laboratories. Aude Billard was also supported in part by a fellowship from the Swiss National Science Foundation.

\section{References}

[1] K. Sing Bing Kang Ileuchi," A robot system that observes and replicates grasping tdss, " in Proceedings., Fifth International Conference on Computer Vision 1995
[2] M.I. Kuniyoshi and Inoue,“ Learning by watching: Extracting reusable tak kowl edge from visual observation of human performance," IEEE Transactions on Robotics and Automation, vol.10, no, Gpp799- 822, 1994

[3] H. Asada, H.; Ixımi, " Automatic program generation from teaching data for the hybrid control of robots," in IEEE Transactions on Robotics and A,utomation 1989 , vol.5 2, pp. 166 -173 .

[4] N. Delson and H. West," Robot programming by human demonstration: adaptation and inconsistency in constrained motion," in IEEE International Conference on Robotics and Automation, 1996, vol. 1 , pp. $30-36$

[5] M. Skubic and R.A. Volz, "Acquiring robust, force-based assembly ki lls from human demonstration," in IEEE Transactions on Robotics and Autonation 000, vol. 16:6, pp.772-7 81 .

[6] I. Kamon, T. Flash, and S. Edelman" Learning visually guided grasping: a test case in sensorimotor learning," in IEEE Transactions on Systems, Man and Cybernetics, Part A 1998, vol. $28: 3$.

[7] M Tomasello, Eul tural transmission in the tool use and communicatory signaling of chimpaxees, " Language and intelligence in monkeys ans apes: comparative developmental perspectives, pp.274- $311,1990$.

[8] L. Berthouze, P. Bakker, andY Kuniyoshi, "Learning of oculomotor control: a prelude to robotic imitation," in Proceedings of the 1996 lEEE/RSJ International Conference on Intelligent Robots and Systems '96, 1996, pp. $376-381$.

[9] A. Billard, "Learning motdxi s lls by imitation: a biologically inspired robotic model," Cybernetics $\mathcal{G}$ Systems Journal, special issue on Imitation in animals and artifacts vol. 32:1-2, pp. 155-193, 2001.

[10] J. Demi ris, Movement imitation mechanisms in robots and humanPh. D. thesis, Dept. of Artificial Intel ligence,Uni versi ty of Edinburgh, May 1999

[11] S. Schaal, s "I mitation learning the route to humanoid robots, "Trends in Cognitive Sciencessol. 3(6), pp 2 33-242 1999 .

[12] S. Schaal, " Learning from demonstration," Advances in Neural Information Processing Sysod.Anspp. 1040-1046, 1997.

[13] M. Arbib, A. Billard, M. Iacoboni, and E. Oztop, “ Mirror neurons, imitation and (synthetic) brain imaging," in Neural Networks (to appe,ai) 000 .

[14] A.J. Ijspeert, J Hallam, andWi llshaw, " Evolving swimming controllers for a simulated lamprey with inspiration from neurobiology," Adaptive Behavior, vol.7, no2 pp. 151-172, 1999

[15] J.J Hopfiel d, "Neurons with graded response properties have collective computational properties like those of two-state neurons," in Proceedings of the National Academy of Sciences vol. 81, pp. 3088-3092. Washi ngton: The Academy, 1984.

[16] F Lacquaniti andJ F Soechting, "Simulation studies on the control of posture and movement in a multi-jointed limb," $B i$ ological Cybernetics vol.54, pp. $\quad 367-378,1986$.

[17] A. Billard and Hayes, "Dama, a connecti onist architecture for control and learning in autonomous robots," Adaptive Behavior, Vol. 7:p.p. 35-64, 1999.

[18] D. T. Lin, P A. Ligomenides, and E. Dayhf, “ Learning spatio-temporal topology using an adaptive time-delay neural netwok, " in Proceedings of World congress on neural networks, Portland, OR 1993, vol. 1, pp29 1-294

[19] S. Schaal, The sl si mulation and real-time control software prage, " Tech. Rep. Gmputer Science Tech Report, http://www-sl ab.usc.edu/publ ications/schaal -TRS , University of Southernal ifornia, 2001. 